\title{
NON-TUBERCULOUS FIBROSIS OF THE LUNG IN CHILDREN.
}

BY

\author{
C. D. S. AGASSIZ, M.D., M.R.C.P., D.P.H. \\ AND
}

W. J. GILL, M.D.

From the High Wood Hospital for Children, Brentwood, Essex.

Since the work of Clark, Hadley and Chaplin was published on "Fibroid Disease of the Lungs" in 1894, a number of papers have been written on small collections of instances of this disease, or on individual cases, but so far no work appears to have been published giving the results of examination of a number of such cases by modern methods, e.g., X-rays, etc. We have been fortunate in having had under our supervision a considerable number of patients suffering from this disease and have investigated these patients by clinical, bacteriological and other methods, and by X-rays, and the results obtained seem worth recording, with a view to obtaining more general recognition of the occurrence of this disease.

Sex. The disease was found to occur equally in the two sexes. This accords with the experience of other writers.

Age. Fibrosis of the lungs may occur in children of any age. Not infrequently it occurs in children of under one year and we have records of cases, diagnosed on clinical evidence alone, under two years of age. Owing to the difficulty of obtaining blood in such cases we have not been able to investigate them as fully as the older children, but the evidence obtained from these older children is sufficient to warrant us in believing that the disease may be diagnosed in infancy.

Of our cases, the incidence was greatest in children from 5 to 10 years of age. Clark, Hadley and Chaplin showed that the incidence of the disease was greatest in patients under 15 years of age. The age incidence noted by these and other writers is rather above what we have stated, but this discrepancy appears to be due to the fact that these writers have included among their records a number of cases of bronchiectasis, which we regard as being more in the nature of a sequela of the disease.

Family History. Examination of the family history shows a marked contrast with that usually obtained in cases of pulmonary tuberculosis in children, where a large percentage show a history of contact with a case of tuberculosis. In fibrosis such a history is exceedingly uncommon. Of 37 cases examined, in only two was there definite history of a member of the family suffering from pulmonary tuberculosis. There appears to be no special familial tendency to fibrosis and in our cases no other members of the family were affected. 
Previous History. Examination of the previous history shows a high incidence of measles, whooping cnugh or pneumonia. The other exanthemata only occurred in a few cases, e.g., scarlet fever (4), diphtheria (2), rubella (1), chicken pox (3), mumps (1), influenza (1), and in one case there was a history of empyema. Presumably the occurrence of these diseases had little bearing on the origin of the fibrosis.

But the number of cases in which there was a history of measles (24) whooping cough (19), and pneumonia (22), is large. The incidence of measles and whooping cough is not above that normally occurring among children, but the incidence of pneumonia is greater. The histories obtained showed that pneumonia occurred either alone or as a sequela of measles or whooping cough. In a further 6 cases there was a history of bronchitis and this figure does not include those cases in which a history of winter cough was given.

The striking fact brought out by this analysis is the high incidence of pneumonia-approximately $60 \%$ of the cases reported.

\section{Symptoms and Physical Signs.}

Cough is the most frequent symptom found. It has been described by some writers as being spasmodic in character and occurring in the morning when the child wakes, but in our experience it is more frequently present in the day time and is rarely spasmodic. The cough varies from time to time. It may be occasional, slight, or absent altogether at intervals ; but if absent it recurs at the onset of any slight catarrh of the lungs, and as these children are subject to catarrhal attacks, particularly in winter, the cough becomes evident or aggravated during the winter months. This aggravation of cough; or alternation of cough with periods of freedom from cough, may persist for long periods. Exertion, even slight, may be provocative of an attack of coughing usually of an irritative type. During the acute exacerbations to which these children are subject the cough becomes frequent and troublesome, but expectoration, if present, is scanty. Clark, Hadley and Chaplin describe vomiting as occurring as a result of a severe paroxysm of coughing, but the description they give appears to refer rather to that of a case of bronchiectasis than of fibrosis, and we have not observed such vomiting except in cases in which bronchiectasis has supervened on a pre-existing fibrosis.

Expectoration is conspicuous by its absence in the majority of patients. This is true of the disease in all stages, except the most extensive, and even then the expectoration is rarely large in amount. In our series the greatest quantity of sputum coughed up in 24 hours by any one patient did not exceed one ounce. The sputum consists of thick, sticky, gelatinous mucus, with possibly some muco-purulent material flaked through it.

As the disease progresses, the muco-purulent character of the sputum becomes more evident. In those cases which go on to bronchiectasis expectoration increases and develops the stale, sickly or fœtid odour characteristic of that disease. The glairy mucus obtained in cases of fibrosis is not offensive, nor fœitid, neither is it nummular. The difficulty of obtaining sputum from children is well-known, and it might be supposed that the explanation of the 
absence of sputum was due to the children's swallowing it. We do not believe that this is a sufficient explanation, however, because children of similar ages, suffering from pulmonary tuberculosis can be taught to, and do, expectorate a quantity of sputum daily. More probably the quantity of secretion is so small as to be almost negligible, and the little there is is so tenacious and sticky that it is only coughed up with difficulty.

The sputum does not contain tubercle bacilli. Recent work has shown the importance of examination of the fæces for tubercle bacilli where no sputum can be obtained. Tubercle bacilli are not found in the fæces of children suffering from this disease.

Dyspnoea. Children whose disease is slight may not suffer from dyspnika. In 40 per cent of our cases there was no complaint or evidence of dyspnoea. In the more advanced stages of the disease dyspnoea on exertion becomes very evident. Slight exertion, such as running up and down a flight of stairs, will produce dyspnœa out of all proportion to the amount of exertion under. taken. In the still more advanced cases dyspnoea may be present to some extent even when the child is at rest, but it is curious to notice that such dyspnoea, though quite evident to the observer, is apparently unnoticed by the child himself. If such a child be asked if he is out of breath he will almost invariably say "No," even though his breathlessness is sufficient to make speaking an obvious effort. That such children do not feel breathless is evident on watching them playing games such as football or netball. They take an active part in such games and do not appear to restrict their exertions in any way, though to the observer they appear markedly breathless. They differ in this way from children suffering from active pulmonary tuberculosis: who will avoid exertion which makes them breathless or tired.

Hocmoptysis rarely occurs. Slight staining or streaking of the sputuin with blood takes place occasionally, but a larger hæmoptysis is of rare occurrence, and if it occurs there seems no tendency for it to recur. Consideration of the pathology of the disease would lead one to expect this, as in fibrosis of the lung there is not that erosion and breaking down of tissues that occurs in pulmonary tuberculosis, and such bleeding as does occur is probably due to rupture of a small vessel in the bronchial mucous membrane.

Pain is seldom present and is never severe. When present it is located in one or other side of the chest, and partakes rather of the sensation of a pulling or tugging on the affected side.

Cyanosis is one of the most constant signs of this disease and in our opinion one to which insufficient attention has been paid by previous writers. In children with extensive fibrosis the lips, cheeks, and tips of the ears are bluish or purple in colour, the surrounding skin being dusky or dark sallow. In leis extensive disease only the lips and cheeks may show a cyanotic tinge, and in still less extensive disease the lips alone may be affected. The intensity of the cyanosis is increased by exercise or cold weather. Children with marked cyanosis as a result of fibrosis of the lungs tend to develop a coarseness of the features which may be such as to suggest the development of slight clubbing, particularly of the tip of the nose. 
One further point regarding cyanosis may be noted here. We have observed that when children, who are suffering from extensive fibrosis of the lungs and are cyanosed, develop tuberculosis, the cyanosis diminishes in degree as the tuberculosis develops. The explanation of this change appears to be found in the pathological changes taking place in the diseased tissue to which we refer more fully below.

Clubbing of the finger tips was found in two-thirds of the cases and varies in degree according to the severity of the disease. In cases of slight disease there may be only slight curving of the finger nails. Cyanosis and clubbing appear to be related. In 75 per cent. of our cases the two signs were present

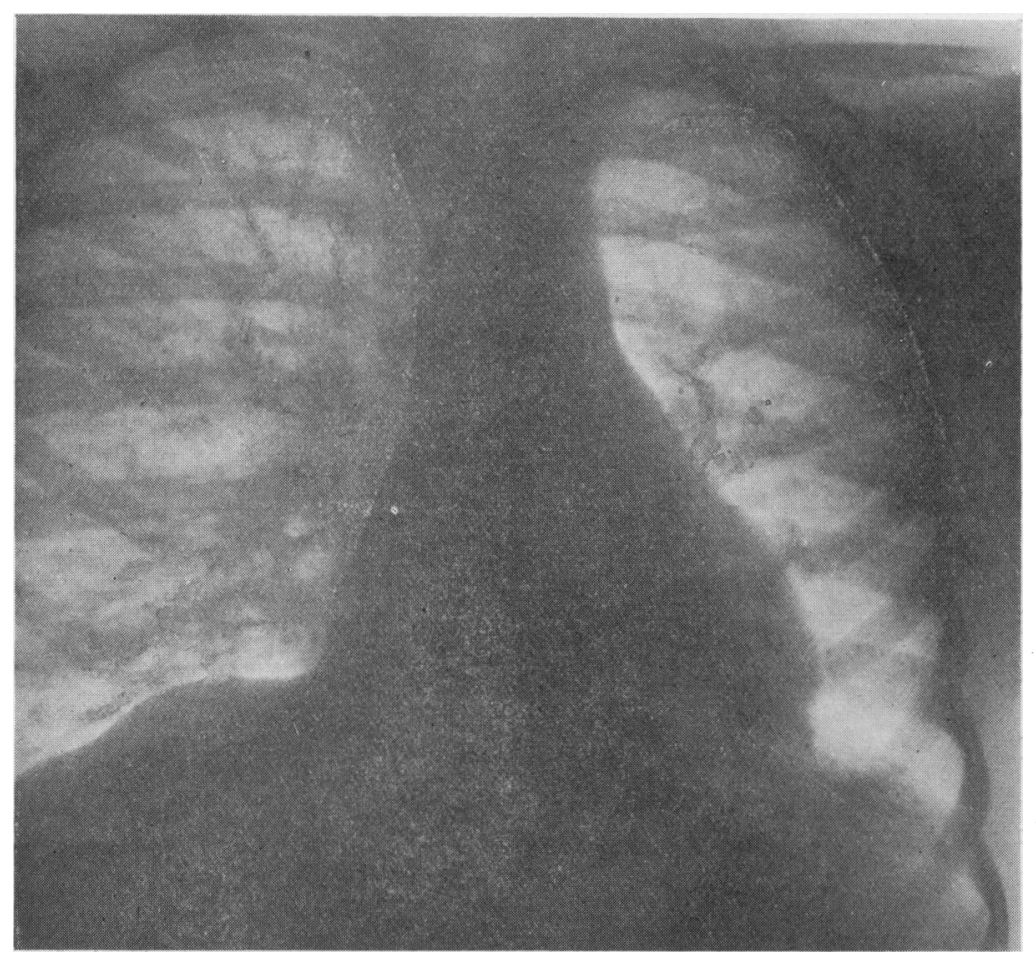

Fic. 1. N.G. Shows apparent bulging of right lung to left side and displacement of heart.

and it was observed that, though cyanosis without clubbing did occur, rarely, if ever, did clubbing occur without cyanosis. The clubbing is more marked than that found in children suffering from pulmonary tuberculosis. Indeed, in our experience, clubbing is not a marked feature of pulmonary tuberculosis in children, except where tuberculosis has been superimposed upon a pre-existing fibrosis of the lungs. In such cases the clubbing tends to diminish with the extension of the tuberculous process.

Appearance and Physique. Children, whose disease is of short duration or slight, may be up to or above the average size for their age ; they may be plump and healthy-looking, a striking feature being the high colour of their cheeks. 


\section{NON-TUBERCULOUS FIBROSIS OF LUNG IN CHILDREN}

In cases of longer duration the cyanosis is more evident, the coarseness of the features already described may be present, and such children are under the average in weight and height for their age. The extremities, particularly the hands, may be bluish even in warm weather. In cold weather the cyanosis of the features and extremities becomes more marked and gives the appearance of the child being "blue with cold," but such children do not complain"of feeling cold nor do they appear to suffer from the cold more than other children. In cases of long standing the physique is poor. Evidence of rickets may be present, particularly in the chest.

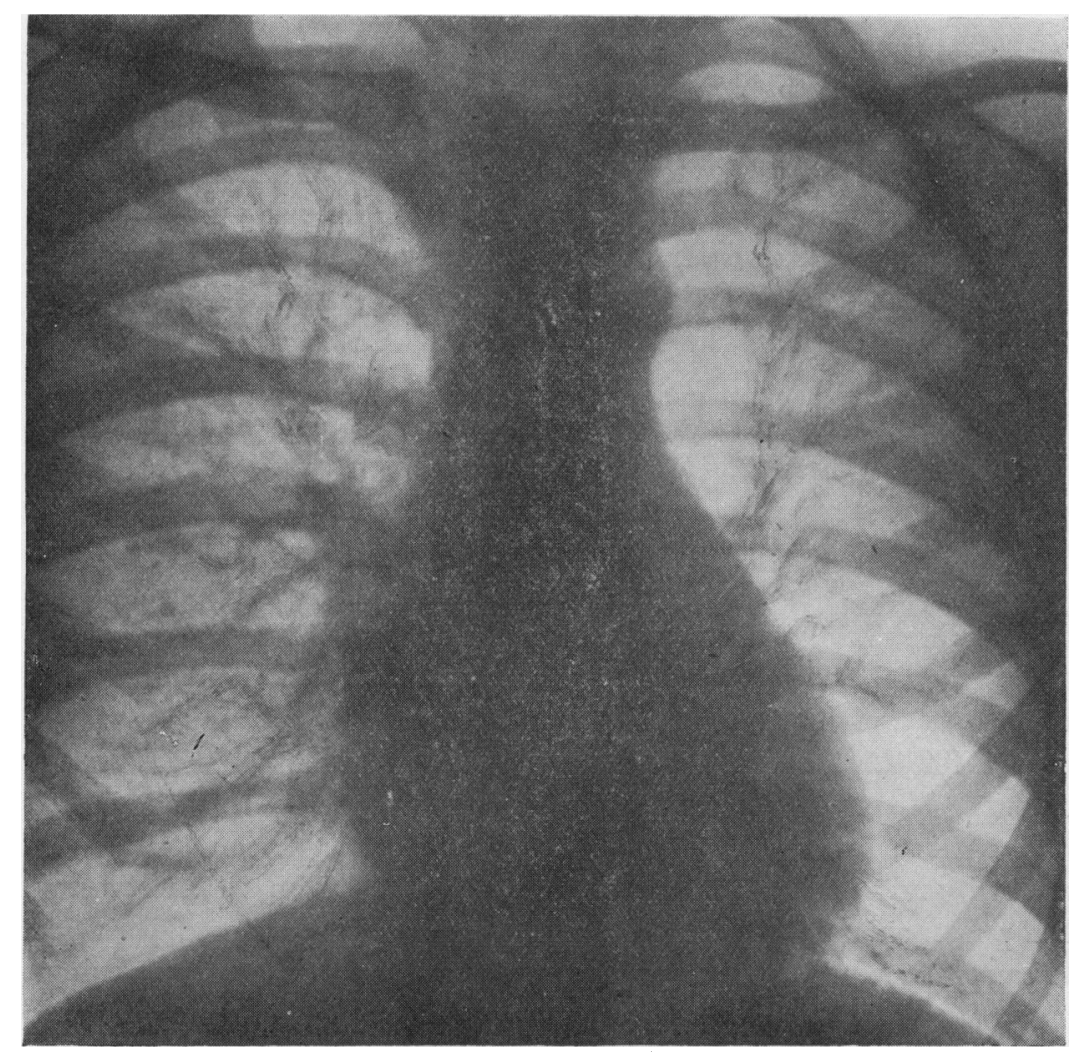

Fig. 2. L.M. Shows increase of root shadows radiating to base and to less extent to upper part of lungs.

Physical Examination of Chest. On deep inspiration there is either general diminution of the expansion of the chest or diminished expansion of one side, and indrawing of the lower intercostal spaces (8th to 10th) may occasionally be seen over the affected side. Displacement of the cardiac impulse is evident where such exists. On palpation there may be no alteration of vocal fremitus, but more usually it is diminished, though never absent altogether. On percussion the note over the affected lung is impaired even in the early stages of the disease, this impairment being most evident at the base of the lung. 
Where the note is impaired over the whole lung, the dullness increases in degree from apex to base, and in this contrasts with that found in cases of pulmonary tuberculosis in children, in which the dullness is most evident at the apex of the lung and diminishes towards the base. Though one lung usually gives evidence of being more affected than the other, the disease is probably always bilateral, and in many cases impairment of note over the whole of one lung is accompanied by impairment at the base of the other lung. The dullness is never so absolute as that found over fluid.

On auscultation there may be no adventitious sounds present, though the breath sounds may be diminished. More usually are found râles of a coarse, dry, possibly leathery character, more evident or more profuse towards the base. Not infrequently crepitations may be heard at the base, giving the impression that they are being produced near the surface of the lung. We regard this type as indicating greater involvement of the pleura. Bronchial breathing may be present opposite the inferior angle of the scapula, and may extend along the vertebral border and be of such intensity as to suggest the presence of a cavity. The extent, frequency, and profuseness of the adventitious sounds vary from time to time. At one examination the whole chest may be filled with loud consonating râles and at the next examination a few days later there may be complete absence of adventitious sounds. So frequently does this occur that we regard it as being characteristic of the disease.

During the pyrexial attacks (described below) very little evidence of bronchopneumonia may be found by auscultation, though $\mathrm{X}$-ray examination shows some consolidation, but there may be an increase of the signs which are present when the child is apyrexial. The râles give the impression of being moister in character or more profuse in extent, but, owing to the presence of moist sounds in the chest when the child is apparently well, it is often extremely difficult to say whether there is any increase in their extent or not. Such cases seen for the first time during a pyrexial attack are sent to hospital as pneumonia. If these attacks recur, the history given is one of recurring attacks of pneumonia; that the chest does not clear between the attacks is overlooked, and thus the true nature of the child's illness is not recognised. Such a pneumonic attack may cause death in a few hours or days.

Temperature. This is usually normal, but certain variations from the normal occur, and are described below. Though there may be no rise above normal, we have noticed that an irregularity within normal limits occurs in the form of a curve over a period of two or three weeks (Chart I).

Citakt I.

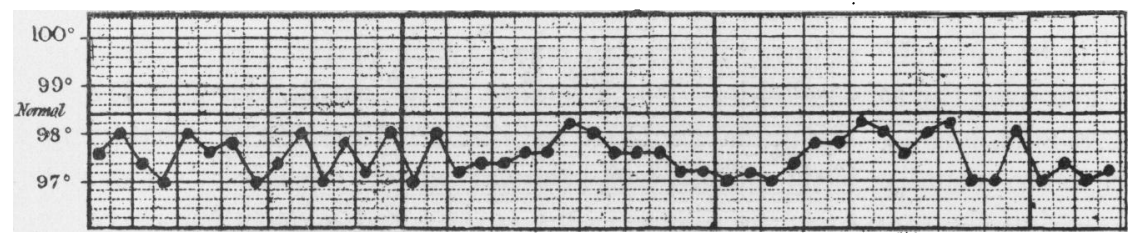




\section{NON-TUBERCULOUS FIBROSIS OF LUNG IN CHILDREN}

The other variations from the normal are as follows :-

The temperature, which has previously been normal, may suddenly rise sharply to $100^{\circ}$ or $101^{\circ} \mathrm{F}$. or higher, and after 24 or 48 hours fall sharply (Charts II and III.) During this period the child appears comparatively well, does not complain of feeling ill, the pulse rate is raised, but the respiratory rate is unchanged, and the child rarely requires to remain in bed for more than a day or two. If an X-ray photograph is taken during this period, evidence of inflammation is found round the bronchi, or a patchy consolidation, usually at the base or near the root of one lung.

Cinart II.

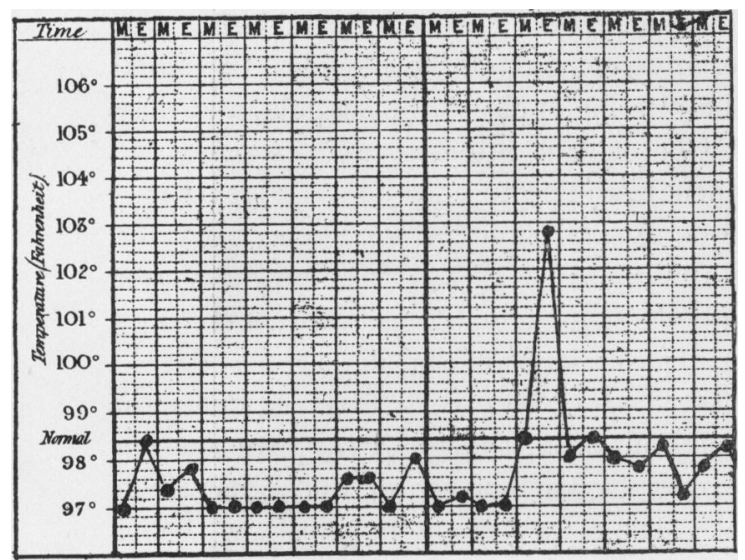

ChakT III.

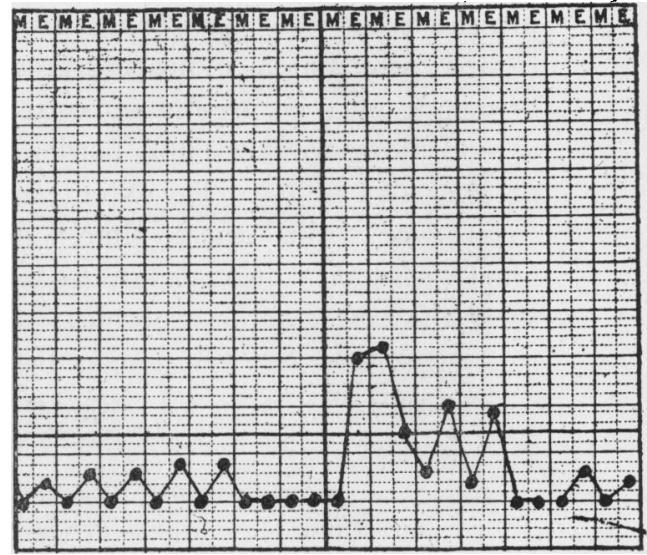

The second variation that may occur is observed as a rule in patients with extensive disease. In this type the temperature rises sharply to $102^{\circ} \mathrm{F}$. or higher and remains raised for some days, usually a week or so, and resembles the temperature curve of lobar pneumonia (Chart IV). During this attack the child looks anxious, the pulse is rapid, the cyanosis becomes extreme, the breathing laboured and wheezy, and the respiratory rate is increased, though not to the same extent as in lobar pneumonia.

Citakt IV

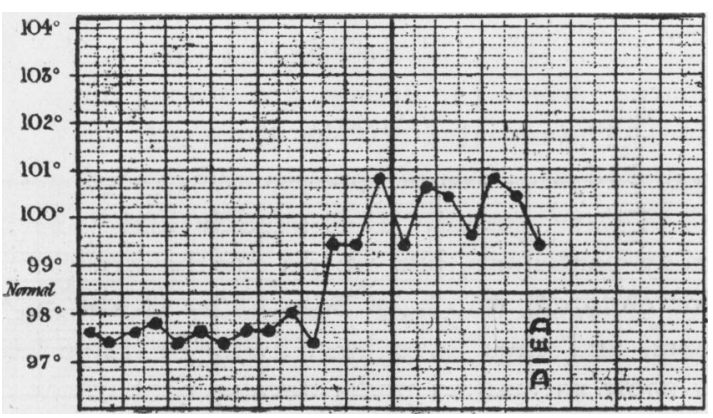


Weight. Only 25 per cent. of our cases were up to, or above, the average weight for their age, and one-third of the cases were $14 \mathrm{lbs}$. or more under weight. This under-development did not appear to be due to the extent of the damage to the lung, for the diminution in weight was as marked in the cases in whom the blood-oxygen content was nearly normal, as in those in whom it was markedly diminished.

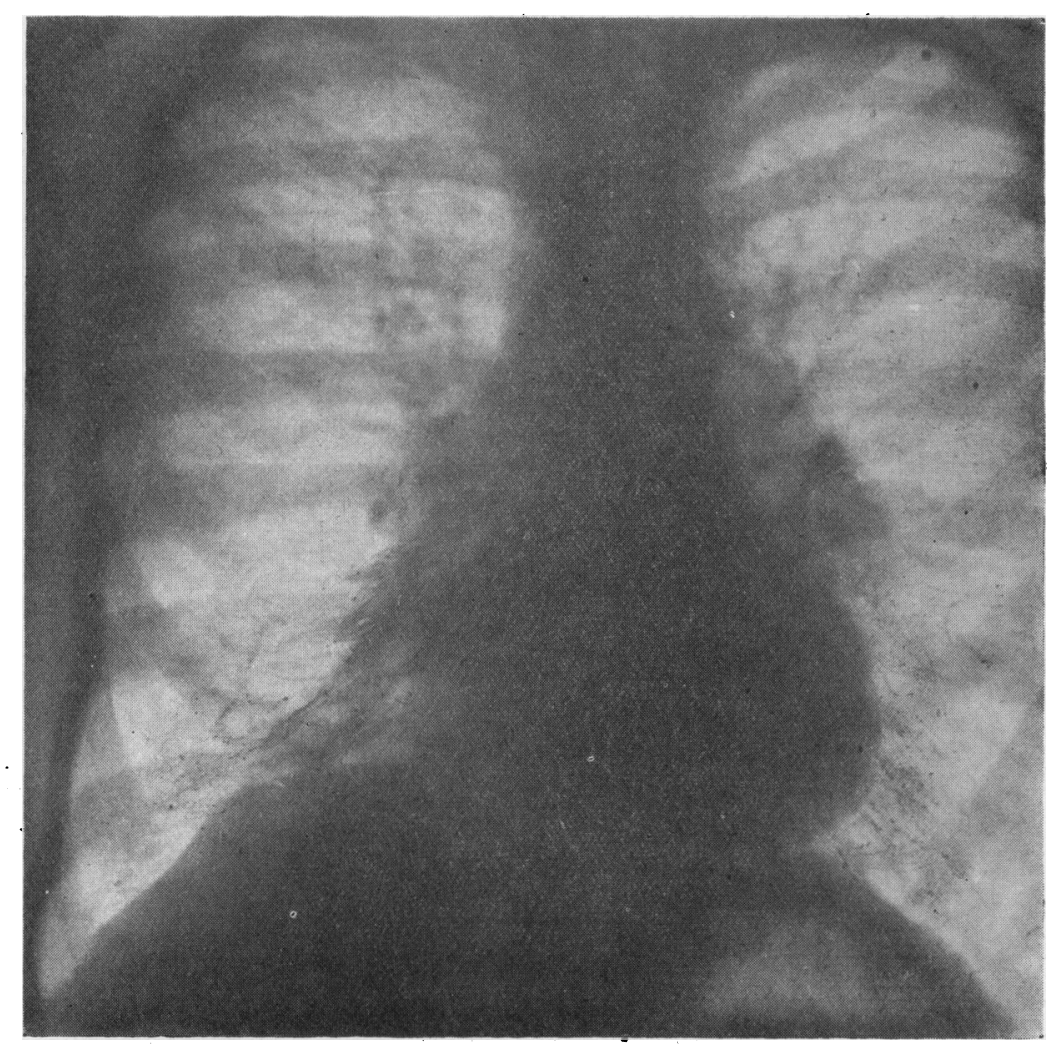

FI(x. 3. R.A. Shows massive root shadows extending particularly to bases.

Examination of the previous history showed that under-development in weight was most marked where the disease was of long duration, though not necessarily extensive. The repeated attacks of catarrh necessitating confinement to the house or bed with loss of appetite and general malaise may help to explain the lack of development. It is possible that bad home conditions or under-feeding may contribute, but we do not believe that these factors fully explain it, for children, who have been under treatment in hospitals or institutions for years, and thus have been living in stable and satisfactory surroundings, may show as marked under-development of weight as children wh o have been living at home. 
Von Pirquet Reaction. In the majority of cases the von Pirquet reaction is negative. In our series of cases 87 per cent. gave a negative result and 13 per cent. ( 5 in 37 ) a positive reaction. The high percentage of negative results obtained is striking and lends support to the view that these cases are not tubercular in origin.

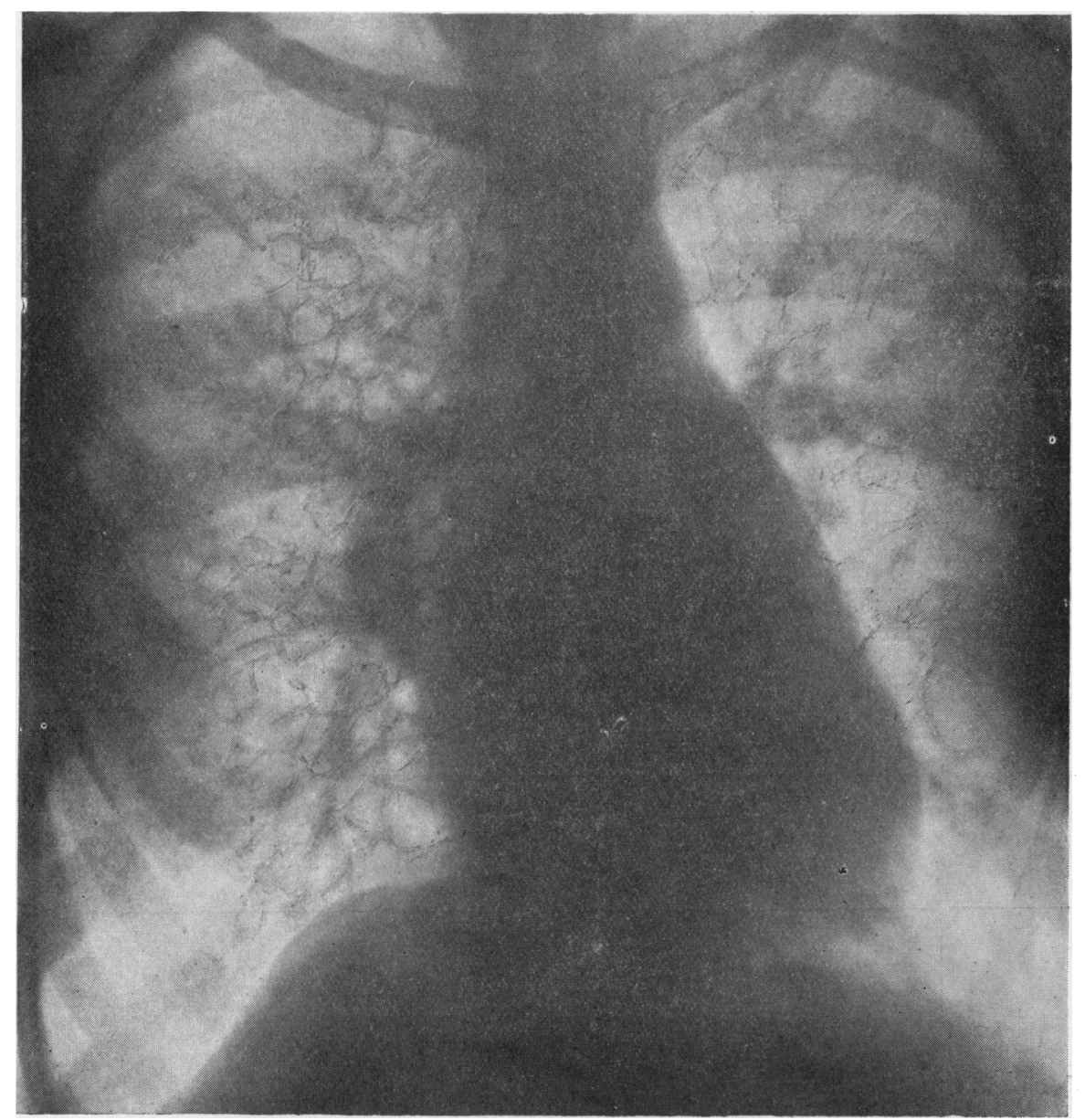

Frit. 4. C. B. Shows honeycomb appearance.

Wassermann Reaction. The possibility of fibrosis being due to syphilis led us to have the blood tested in all casss. In the majority the results were negative, but 8 per cent. ( 3 in 37 ) gave a positive result. The patients with a positive reaction did not appear to differ in any way, either in history, physical signs, symptoms, or radiologically, from those in whom the test was negative. Anti-syphilitic treatment did not appear to have any effect on the disease. The percentage of positive results obtained, however, suggests that syphilis may be a contributory cause of the disease, and further investigation of this point is necessary. 
Blood-Oxygen Content. Lundsgaard and Van Slyke have shown that cyanosis develops as the result of want of oxygenation of the blood, and that when the oxygen unsaturation rises above $6 \cdot 7$ volumes per cent. in the capillary blood, signs of cyanosis develop. The frequency with which cyanosis occurs in children suffering from this disease led us to investigate the bloodoxygen content in the hope that we might obtain additional information which would be of use in diagnosis.

Venous blood was taken for this purpose. The blood-oxygen content was found to be diminished in every case. It was usually most diminished where the cyanosis was most marked, but even in cases in which cyanosis was slight the blood-oxygen content was diminished as compared with the normal. In some cases a blood oxygen content of $6 \cdot 7$ volumes per cent. was observed. The diminution of blood-oxygen appeared to depend on the extent of the fibrosis rather than on the length of history of the case.

As a control to these observations blood was taken from children in whom no disease could be detected, and in these the blood-oxygen content was found to be normal. In order to compare the above findings with those obtained in pulmonary tuberculosis, the blood of children suffering from slight but active pulmonary tuberculosis, in whom the diagnosis had been confirmed by finding tubercle bacilli in the sputum, was examined. In all these cases the bloodoxygen content was normal. In cases of pulmonary tuberculosis of long standing, which we believed to be examples of tuberculosis supervening on a pre-existing fibrosis, the blood-oxygen content was diminished.

TABLE :

Typical Blood-oxygen Contents in Different Groups.

\begin{tabular}{|c|c|c|c|c|}
\hline & & Disease. & & $\begin{array}{l}\text { Blood-oxygen content } \\
\text { (vols. per cent.) }\end{array}$ \\
\hline N.M. & $\cdots$ & Extensive fibrosis of both lungs & . & $6 \cdot 81$ \\
\hline L.B. & $\ldots$ & Extensive fibrosis of both lungs & . & $6 \cdot 01$ \\
\hline A.W. & $\cdots$ & Fibroid signs at both bases & $\cdots$ & $11 \cdot 88$ \\
\hline E.F. & $\cdots$ & No evidence of disease & $\ldots$ & $12 \cdot 80$ \\
\hline E.W. & $\ldots$ & Pulmonary tuberculosis & . & $12 \cdot 40$ \\
\hline W.C'. & $\cdots$ & Pulmonary tuberculosis ('T.B. $\mathrm{i}$ ) . & .. & $13 \cdot 40$ \\
\hline J.C. & $\cdots$ & $\begin{array}{l}\text { Pulmonary tuberculosis suparim io oued } \\
\text { fibrosis (T.B. }+ \text { ) }\end{array}$ & on & $8 \cdot 23$ \\
\hline E.C. & $\cdots$ & $\begin{array}{l}\text { Pulmonary tuberculosis superimposed } \\
\text { fibrosis (T.B. }+ \text { ) }\end{array}$ & on & $9 \cdot 21$ \\
\hline
\end{tabular}


At this stage it may be worth while considering shortly the pathological changes that take place in pulmonary tuberculosis and in fibrosis of the lung, in order to explain the different blood-oxygen contents in the two diseases.

In fibrosis of the lungs the fibrosis occurs around the bronchi, in the interlobular septa, and in the alveolar wall. As a result of the involvement of the alveolar wall the absorption of oxygen from the alveoli is interfered with, though the circulation of blood through the capillaries surrounding it is maintained. The result is that the blood returning to the heart from the area of the lung affected is un-aerated and thus is produced what Lundsgaard and Van Slyke term an "un-aerated shunt." The proportion of blood affected varies with the extent of lung involved, and when more than one-third of the blood is involved the drop in the oxygen content is sufficient to produce cyanosis.

In tuberculosis, on the other hand, though the tubercle may form in the alveolar wall and may interfere with the normal function of the alveoli to some extent, it does not produce an "un-aerated shunt" because the blood vessels in the tuberculous deposit become thrombosed and, circulation through them being thus prevented, the blood is diverted through other parts of the lung and is oxygenated.

We noted that when a cyanosed patient suffering from fibrosis develops tuberculosis, the cyanosis diminishes in degree with the increase of the tuberculous process. The explanation of this would appear to be that when tuberculosis develops in the area of fibrosis the blood vessels in the tuberculous deposits become thrombosed, and the blood supply is thus cut off from this area and diverted through the healthy parts of the lung. As the tuberculosis spreads through the fibrosed lung, the area of the shunt becomes reduced and the cyanosis diminished. If these arguments are correct, the examination of the blood-oxygen content should give the following results :-

1. In fibrosis the blood-oxygen content should be diminished.

2. In tuberculosis the blood-oxygen content should be normal (except in massive infection of the lung).

3. In fibrosis in which tuberculosis developed, the blood-oxygen content should increase with the increase of the tuberculosis.

4. In tuberculosis which was healing, i.e., fibrosing, the blood-oxygen content should decrease as healing advanced.

We have already dealt with the first two points. With regard to the third and fourth we have so far not had an opportunity of making a sufficient number of observations to be of value.

$X$-ray Examination. On screen examination the apices light up well and equally; there is some increase of root shadows extending particularly towards the base ; and there may be irregularity of the outline of the diaphragm on deep inspiration, and blurring of the cardio-phrenic angles. There is no evidence of enlargement of the bronchial glands. 
Radiograms may show some deflection of the trachea to one or other side. Occasionally there appears to be some bulging of the less affected lung towards the opposite side, suggesting the pushing over of the mediastinum, but whether this deflection is due to a pulling over as a result of the fibrosis of the lung, or to a pushing over from emphysema of the less affected lung, is not yet clear, but it seems probable that it is in the nature of a pulling over as a result of contraction of the lung. The heart shadow may be unaltered in position or may be displaced. The shadows at the root of the lung are increased in extent. In many cases these are dense and massive, extending from the

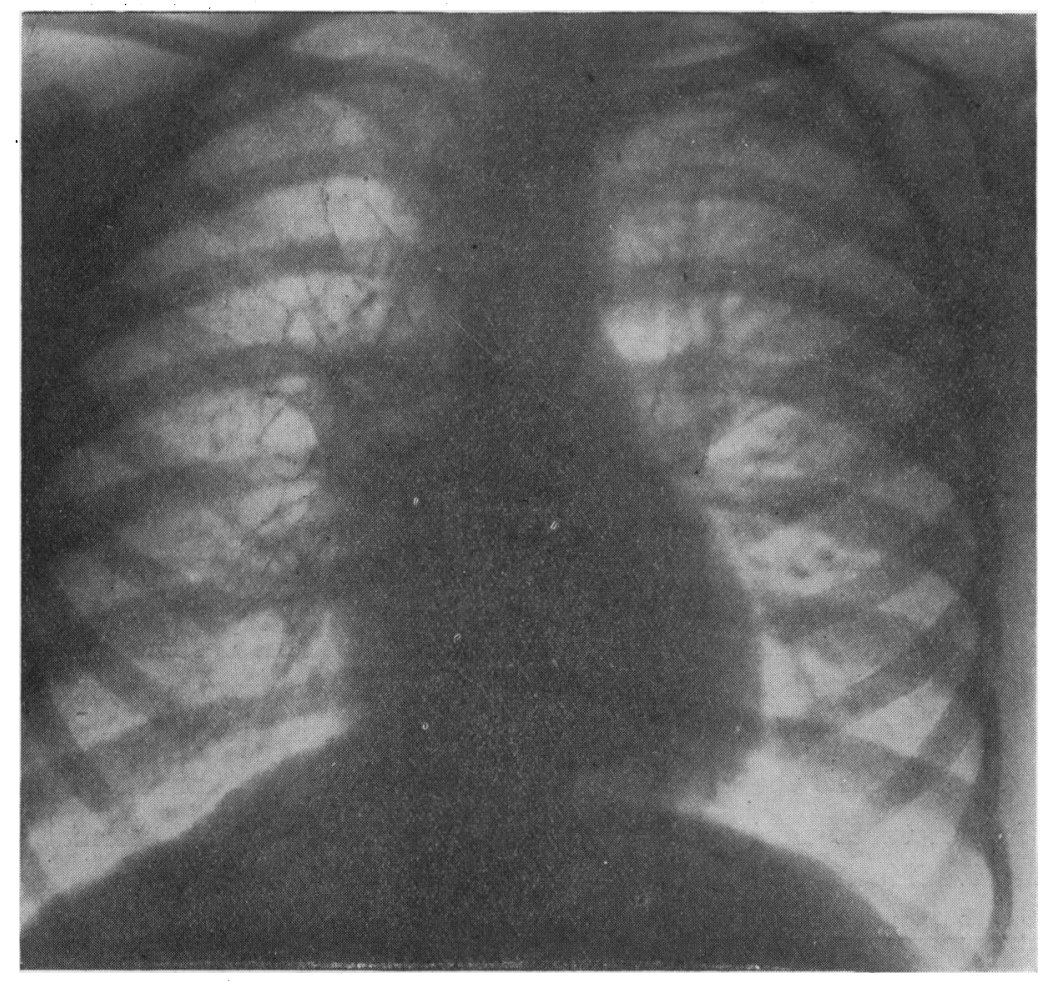

Fic. 5. C. L. Shows star-shaped shadows centred round a bronchus.

level of the third to the sixth or seventh rib anteriorly; and from the central shadow there radiate out into the surrounding lung heavy, streaky shadows most evident towards the base, though at times radiating shadows may be seen extending to the upper part of the lung. They are not usually marked in the middle portion of the lung. The normal reticular formation may be more evident than usual and in some cases a markedly honeycomb appearance, particularly of the upper part of the lung, may be present. Star-shaped shadows, centred round what appears to be a small bronchus seen in cross section, may sometimes be observed. The apices are clear. 
Irregularity of the diaphragmatic outline is of frequent occurrence, and may be present when the root and basal shadows are not marked. The cause of this irregularity is an interesting point, and we believe that it may be explained as follows :-

The lung is most fixed at its root, the outer portions contracting and expanding with respiration. When fibrosis occurs it is found around the bronchi and in the alveoli and interlobular septa, and as a result, of it there is a diminished expansibility of the portion of the lung affected. The diaphragm is retained in apposition with the base of the lung by the negative pressure in

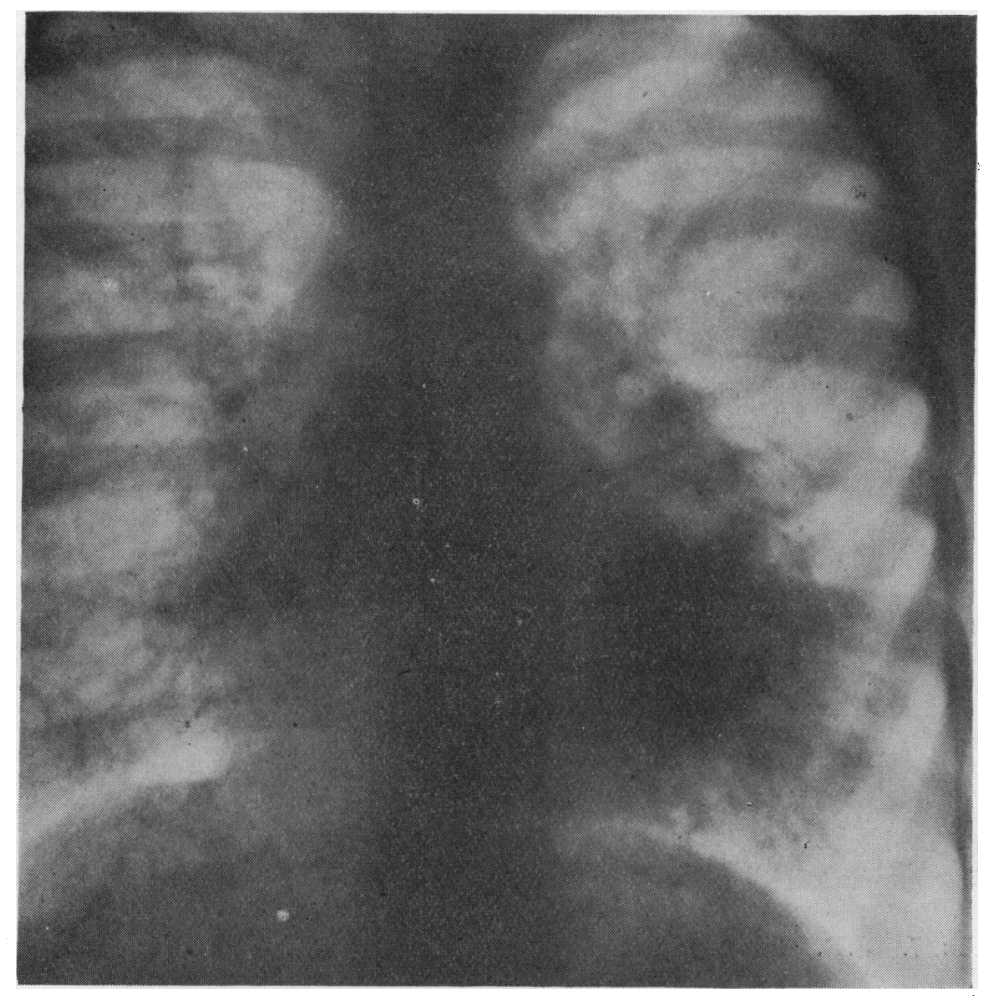

Fig. 6. R.A. Same case as Fig. 3, showing dark shadows which may be found during a pyrexial attack.

the pleural cavity. Now, if fibrosis occurs in the lung around the bronchi extending to the base, the elasticity of this portion of lung is reduced, and the root of the lung being the most fixed point, the area of lung, and consequently the diaphragm, at the lower part of the fibrosed area, will move less on inspiration than the surrounding lung tissue and in consequence irregularity will show in the diaphragm. These irregularities of the diaphragm are invariably present in its inner two-thirds as would be expected if the explanation given above is the correct one. It seems improbable that such irregularities are due to adhesions. If they were, one would not expect to find an irregularity 
on deep inspiration, as the elasticity of the lung would not be affected, and further, there seems to be no reason why adhesions should be restricted to the inner part of the diaphragm.

Though the increased root and basal shadows may be more evident in one lung, there is usually some increase in the other lung. Radiograms taken during a pyrexial attack may show dark shadows near the root or base of the lung.

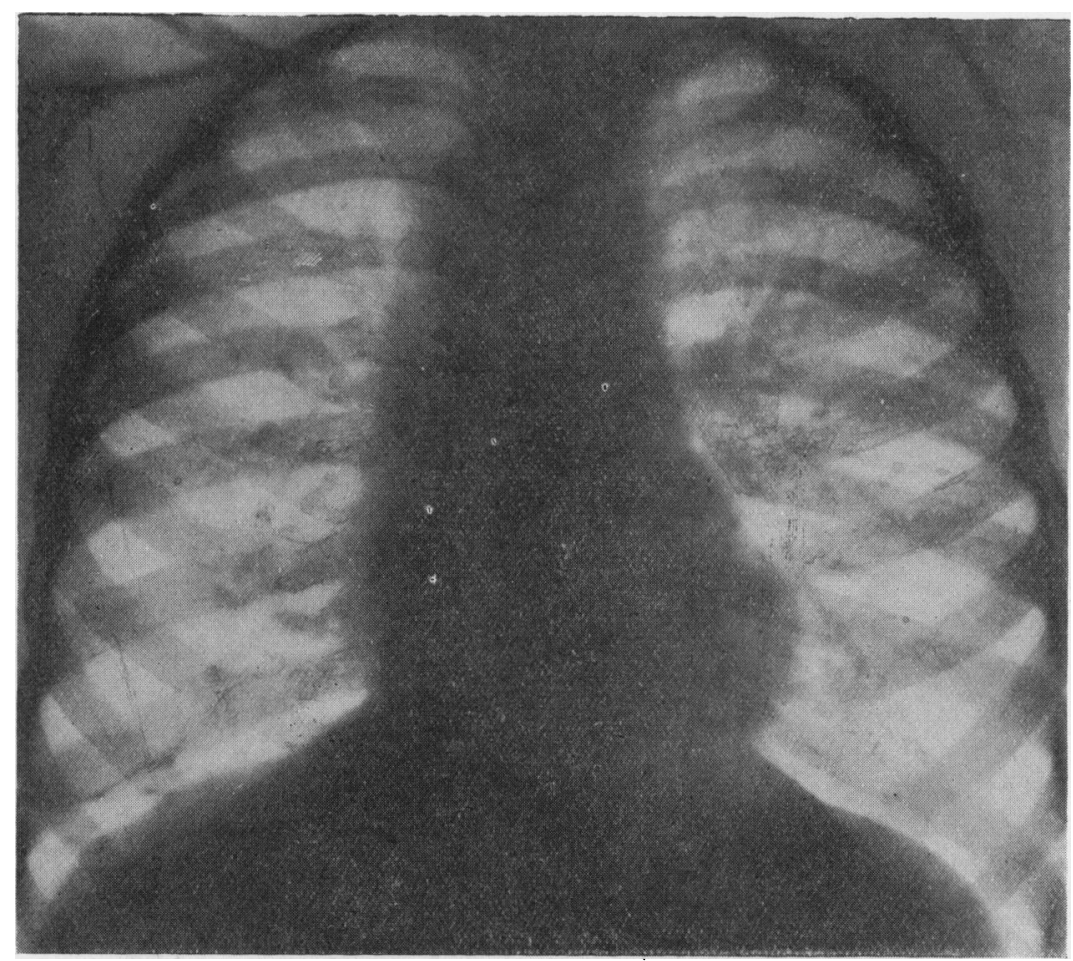

Fir. 7. J.R. Showing thickening round bronchi during a oitarrhal attack.

\section{Complications.}

The subjects of fibrosis appear to be more liable to complications of a septic nature than other children. They frequently show evidence of antecedent rickets. Otorrhœa is not uncommon and such minor forms of infection as tonsillitis, impetigo, and catarrhal affections of the lung occur more often. The bronchopneumonic attacks to which these children are subject have already been described.

Clark, Hadley and Chaplin noted the occurrence of albuminuria in their cases but we have not been able to confirm this. Possibly the fact that they were referring to adults in whom the disease was of long standing, or in whom bronchiectasis was developing, may explain this. 


\section{Diagnosis.}

The diseases from which fibrosis has to be distinguished are particularly :(1) Tuberculosis ; (2) Chronic bronchitis ; (3) Pleural effusion; (4) Thickened pleura; and (5) Bronchopneumonia.

1. Tuberculosis. The importance of distinguishing between this disease and pulmonary tuberculosis is evident, but that it is not appreciated sufficiently is shown by the fact that the cases upon which this paper is founded were sent to hospital as cases of pulmonary tuberculosis. Consideration of the following factors is usually sufficient to distinguish the two diseases.

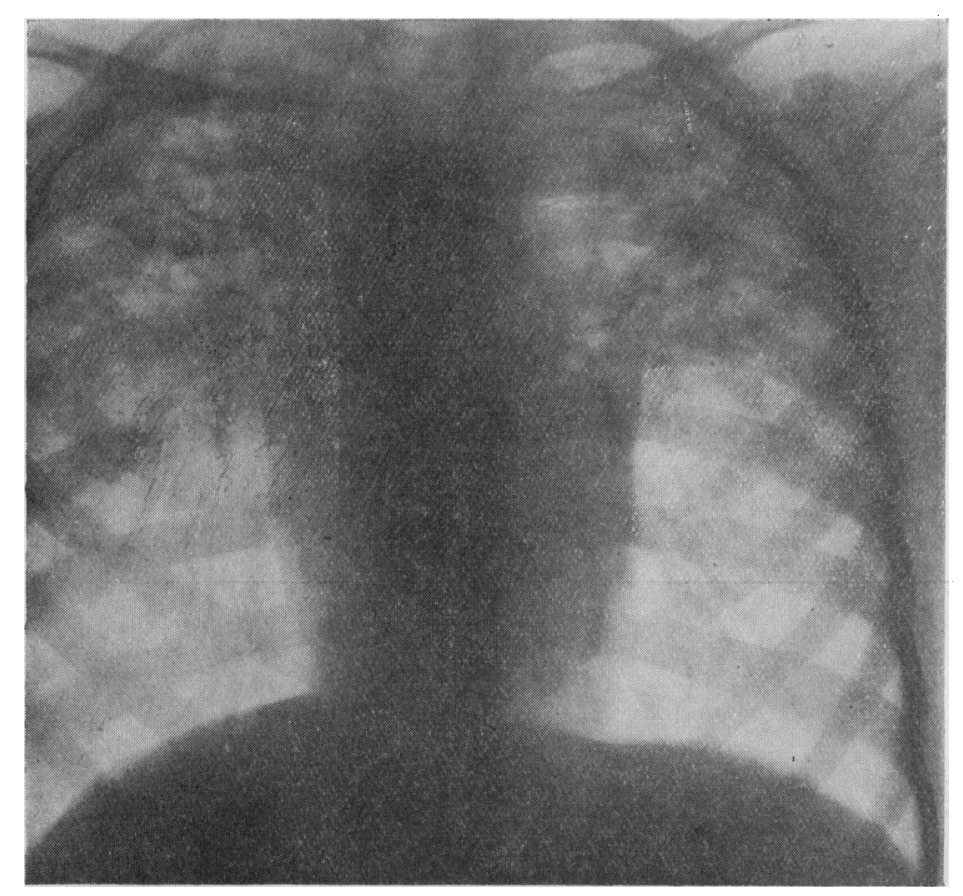

Frit. 8. I.B. Shows typical fibrosing pulmonary tuberculosis.

In the majority of cases of fibrosis there is a history of an attack of pneumonia, either unaccompanied, or as a sequela of measles or whooping cough. There may be a history of repeated attacks of pneumonia. In others the history is one of repeated attacks of bronchitis. In pulmonary tuberculosis there is frequently a history of contact with a case of pulmonary tuberculosis, but in fibrosis such a history is not common.

The characteristic aspect has been described, and is in contrast to that of children suffering from pulmonary tuberculosis.

Children suffering from pulmonary tuberculosis are commonly pale; they are not under-developed unless their disease is of long standing or progressing, and, as they recover, they gain weight rapidly, and usually reach the average weight for their age in a comparatively short time. The symptoms, physical 


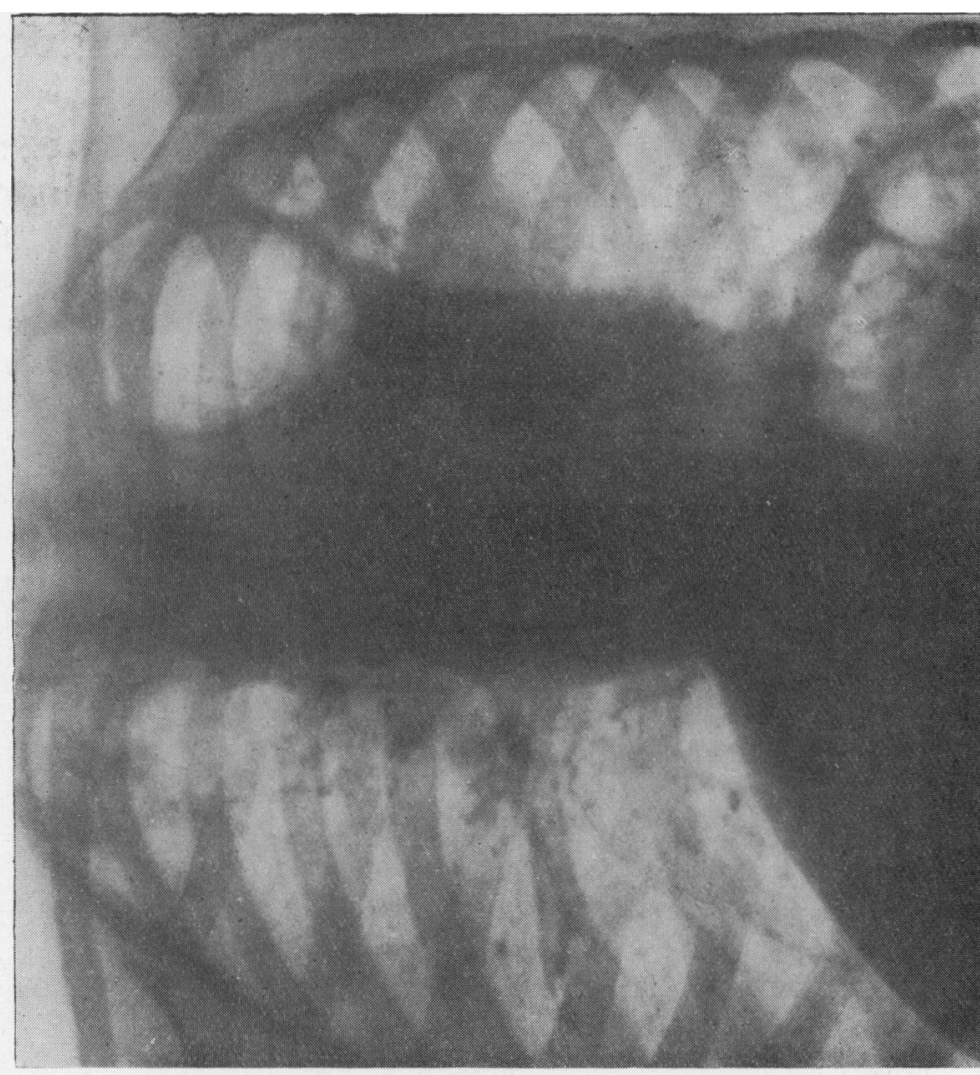

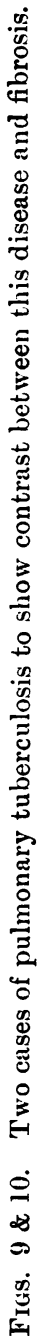


signs, X-ray examination, examination of sputum and fæces, Wassermann reaction, and blood-oxygen content all give evidence which must be considered in attempting to arrive at the correct diagnosis.

The X-ray photograph in pulmonary tuberculosis shows mottling or woolliness more marked at the apices. Cases in which the tuberculosis is healing or healed show, in addition to this woolly mottling, shadows of irregular density particularly near the apex.

The diagnosis from hilum tuberculosis is one which causes more difficulty, but there are a few points which seem to us to warrant the belief that what we have described is not hilum tuberculosis. Many of these cases begin in infancy. On examination of such a case are found numerous râles at both bases, but the X-ray photograph only shows an increase of root shadows. It is difficult to believe that an infant with extensive signs, but apparently in good health, and with little evidence of disease in the lungs as shown by $\mathrm{X}$-ray photographs, is suffering from tuberculosis, which is admittedly a most fatal disease in infants. To older children the same argument applies though to a less extent. In these children it is characteristic to find extensive physical signs, but an X-ray picture which shows remarkably little change. In tuberculosis, on the other hand, the X-ray picture usually shows evidence of far more extensive disease than is given by physical signs. Further, in pulmonary tuberculosis the signs are more evident at the apex, whereas in fibrosis they are more evident at the base of the lung.

It may be argued that the picture we have described is one of so-called 'hilum tuberculosis' in which the disease has gradually become quiescent, and that as a result fibrosis has taken place in the peribronchial areas, producing the typical $\mathrm{X}$-ray picture.

Such an explanation is at first sight plausible, but on reflection it cannot be inaintained. In the first place it makes no attempt to explain the occurrence of cyanosis. Secondly, if healing of the tuberculosis is taking place in the peribronchial areas, the condition of the child should improve. The history of cases of fibrosis is, however, that of a slowly progressive disease. Again, the strongest argument against this theory is the existence of cyanosis with, in consequence, a lowered blood oxygen content. The presence of cyanosis in itself shows that at least one-third of the blood has not been oxygenated. Now, if the fibrosis is the result of a healing tuberculosis, the tuberculous deposits must have been in the alveolar wall as well as in the peribronchial tissue in order to produce interference with oxygenation, so that at least a large portion of the lung area must be affected by a fibrosing tuberculosis which, in contrast to cases of pulmonary tuberculosis, confirmed by finding tubercle bacilli in the sputum, shows no sign in a radiogram except in the peribronchial tissue. Such a proposition cannot be seriously maintained.

(2) Chronic Bronchitis. In distinguishing between chronic bronchitis and fibrosis of the lung the following points should be borne in mind. Chronic bronchitis tends to produce emphysema and evidence of this should be sought for. The percussion note is not permanently impaired, and there is no 
displacement of organs. Contraction of the chest on the affected side, displacement of organs towards the affected side, impairment of percussion note, cyanosis and clubbing of the finger-tips should suffice to distinguish the two diseases.

(3) Pleural Effusion. The diagnosis between fibrosis of the lung and pleural effusion is not difficult. The distinguishing points are that in pleural effusion the dullness is more wooden, vocal fremitus may be absent, and displacement of the organs is away from the affected side. In contrast to this in fibrosis there is not a complete absence of vocal fremitus, and any displacement of organs is towards the affected side. The X-ray picture in the two diseases is distinctive. The use of the exploring needle would settle a doubtful case.

(4) Thickened Pleura. A diagnosis between fibrosis of the lung and thickening of the pleura is not one of-much practical importance. In extensive fibrosis the pleura is probably always thickened, and in thickening of the pleura which follows on pleurisy or pleural effusion there may be a pleurogenous fibrosis. Apart from this the main point of difference is that in thickening of the pleura per se adventitious sounds are absent at all times. X-ray examination may show some diminished translucency on the affected side.

(5) Bronchopneumonia. In the acute stages it may be difficult to distinguish the diseases, but evidence of clubbing of the finger-tips or displacement of the heart will indicate the true cause of the bronchopneumonia. In cases of fibrosis the persistence of the physical signs when the child is convalescent serves to bring out the true character of the disease.

The diagnosis from such rare diseases as sarcoma of the lung, cysts, etc., is not of much practical importance owing to their rarity, but apart from the physical signs X-ray examination should be sufficient to eliminate the diagnosis of fibrosis in most cases.

\section{Prognosis.}

From a practical point of view it may be argued that a diagnosis of fibrosis of the lung is not of much importance, but as we believe that this disease is more common than is generally believed, and less frequently recognized than it should be, and as so many of these cases are labelled and treated as tuberculosis, from an economic point of view at least it merits attention.

The prognosis in these cases is vastly different from that of tuberculosis. Fibrosis of the lung is probably a slowly progressive disease, but the subjects of it may live many years and lead active and useful lives. They are subject to catarrhal attacks and may develop broncho-pneumonia which may cause death. A certain number appear to develop tuberculosis, but in such patients we find that the tuberculous process is of slow growth and, in contrast to children in whom pulmonary tuberculosis develops but is not superimposed on a pre-existing fibrosis, they may live for a number of years, e.g., six, eight or more. Other cases develop dilatation of the bronchi and become bronchiectatic. Possibly in some cases the signs may disappear but so far we have not been able to collect sufficient evidence to say whether this occurs or not. Owing to their susceptibility to septic infections the onset of such infections 
may terminate these cases. In one case (not included in this series) infective endocarditis followed on fibrosis resulting from injury, and in this case we were able to confirm the diagnosis of both diseases at post-mortem.

One further point in connection with this disease is worthy of remark, as it does not appear to have been noted by other writers. We have frequently observed that the subjects of this disease take anæsthetics badly, even such anæsthetics as nitrous oxide, and it is within our knowledge that cases operated on for such diseases as appendicitis have collapsed and died shortly after the operation from no apparent cause. We consider that if attention were directed to this point valuable information. might be obtained.

\section{Treatment.}

Very little need be said regarding treatment, as it is mainly palliative The ordinary open-air treatment in convalescent homes, or sanatoria for children, suits these children very well.

Prevention, so far as possible, of the onset of catarrh should be practised. With the onset of any fœtor of the breath, creosote in increasing doses should be given, and children of tender years will take up to half a drachm, thrice daily, without complaint. The possibility of treatment by vaccines suggests itself, but we have had no experience of their use.

\section{BIBLIOGRAPHY.}

Atkinson, D. W., Amer. J. Med. Sc., Philad., 1925, CLXX, 693.

Bissell, F. S., Amer. J. Roentgenol., N.Y., 1925, XIII, 126.

Carroll, W. F., Gibson, C. B., Banyai, J. A., Amer. Rev. Tuberc., N.Y., 1925, II, 40.

Clark, A., Hadley, W. J., \& Chaplin, A., Fibroid Diseases of Lung, Lond., 1894.

Davis, K. S., Radiology, Minn., 1924, III, 150.

Duhem, P., Arch. de méd. enf., Paris, 1921, XXIV, 663.

Dumarest, F., Ann. de méd., Paris, 1922, T., XI, 219.

Field, C. G., Amer. J. Med. Sc., Philad., 1920, CLIX, 442.

Hebert, G. T., Lancet, Lond., 1922, i, 1145.

Jermain, M. L. F., Tice's Practice of Medicine, 1923, V, 534.

Kahn, M. and Sloan, M. F., Radiology, Minn., 1925, IV, 198.

Lyter, J. C., Med. Clin. N. Amer., Philad., 1920, IV, 819.

Lungsgaard, C., and Van Slyke, D.D., Medicine, Balt., 1923, II, 1.

Mills, H. P., and Watkins, W. W., Radiology, Minn., 1925, IV, 178.

M'Neil, C., Edin. Med. J., Edin., 1925, XXXII, 107.

M'Neil, C., Trans. Med. Chir. Soc., Edin., 1924.25, XXXIX, 152.

Overend, W., Tubercle, Lond., 1920, I, 547.

Palasse, M., Lyon Méd., Lyons, 1925, CXXXV, 46.

Palasse, M., and Badolle, Ibid, CXXXVI, 691.

Riviere, C., St. Bart. Hosp. Rep., Lond., 1905, XLI, 123.

Sante, L. R., Radiology, Minn., 1924, III, 128.

Tallermann, K. H., Arch. Pediat., N.Y., 1925, XLII, 176.

Young, F. H., Charing Cross Hosp., Gaz., Lond., 1926, XXVI, 139. 